\title{
Contextual details, cognitive demand and kinematic concepts: exploring concepts and characteristics of student-generated problems in a university physics course
}

\author{
Javier Pulgar $^{1,2}$, Alexis Spina ${ }^{1}$, Carlos Ríos ${ }^{3}$ and Danielle Harlow ${ }^{1}$ \\ ${ }^{1}$ Gevirtz Graduate School of Education, University of California Santa Barbara, CA, USA, 93106-9490 \\ ${ }^{2}$ Departamento de Física, Universidad del Bío Bío, Avda. Collao 1202, Concepción, Chile, 403000, and \\ ${ }^{3}$ Departamento de Enseñanza de las Ciencias Básicas, \\ Universidad Católica del Norte, Larrondo 1281, Coquimbo, Chile
}

Student-generated physics problems are a way to encourage students to utilize concepts and to identify situations in which to use them. This study looks at three undergraduate physics sections at a university in Chile and how students generated and established their own problems in circular motion (CM) for high school students. By collecting and analyzing the work created by undergraduate student groups, we were able to identify the various physics concepts and characteristics students used when developing the situation of each problem, the content involved, and the question items designed for high school students. Through a description of the concepts and characteristics, we identify differences and similarities regarding kinematic concepts and problem characteristics across the three sections that undergo different sets of physics problems. This work may further our understanding of how students interpret and use the physics ideas taught at the undergraduate level, and the types of problems they are most familiar with depending on instruction. 


\section{INTRODUCTION}

This study asked undergraduates to generate their own problems as an alternative to solving more traditional textbook physics problems, which is a common practice in physics courses at universities in Chile. Creating problems involves additional cognitive challenges associated with generating assumptions and making decisions [1], which is strongly linked to solving real-world problems and creative practices [2].

This study looked at the diverse characteristics that emerged from kinematics problems generated by student groups from three sections in a undergraduate physics course. Problem characteristics include the physics concepts used, types of information included as data, algebraic steps required to solve the problem, contextual details, and the cognitive demand. By analyzing the various problems generated, we evidenced familiarity with the use of concepts and characteristics, possible misconceptions, and speculated the possible cause of observed differences across sections on the overall elaboration of the problems.

\section{POSING PROBLEMS FOR PHYSICS EDUCATION}

Problem posing, according to Jonassen's definition [3] is in itself a type of ill-structured problem, as students are faced with the task of creating solutions, which requires intense dialogue between the groups to engage in decision-making. While working in groups, students must also draw on social skills, a key aspect of collaborative activities. The ways in which students depict their physics ideas through developing problems becomes a valuable opportunity for assessing learning and conceptual development $[4,5]$.

The first element that facilitates creative outcomes in the face of problem posing would be domain-relevant knowledge and skills [6], which, in this context, take the form of a wellstructured conceptual understanding in physics [7]. The literature in learning and creativity has suggested that individuals' conceptual responses depends on whether the information is well-connected to other ideas they are already familiar with. Whether new information is well-connected to prior knowledge influences whether they use novice or expert-like strategies when solving problems [4, 8]. According to the semantic network models [9], memory and knowledge are distributed in such a way that well understood information will be strongly and closely connected in the cognitive network. Consequently, ideas for creating problems are likely to emerge from dense sections of this conceptual network, rather than isolated nodes. This was found in the context of brainstorming sessions, where ideas associated with dense sections of the network would come up faster, followed by less active or a less dense cluster of concepts [10]. This process will continue until there are no more active network areas from where to retrieve ideas. Because first ideas tend to come from denser structures and are better known, teams tend to choose them over ideas suggested at later stages of the task [10].

The second important condition for a successful outcome in problem posing consists of individuals and/or teams engaging in creative-relevant processes [6]. These processes are linked to creative personalities conducive to risk taking for generating new ideas. In the face of an ill-structured activity such as problem posing, creative-relevant processes may encourage students to deviate from conventional ideas (e.g., problems solved during class, textbook exercise), and venture into more novel routes. One of the multiple challenges of solving creative tasks lays on generating the needed assumptions, or constraining conditions, that would pave the route to the final design [1]. We understand the latter as one of the many creative-relevant processes suggested by Amabile [6], more frequently seen in experts than novice physics problem solvers, likely due to differences in prior experiences [1]. With this information it is fair to suggest that exposure to tasks such as problem posing in physics classrooms might facilitate performance on creative activities, as these would increase students' experience in generating ideas, in addition to justification skills, attitudes towards science, regulation of problem solving strategies and information section, all of which are proven to be positive and significant predictors when solving ill-structured activities [7].

Student-generated problems can reflect their conceptual understandings, as well as the different scenarios under which they understand physics concepts, as a proxy of their experiences. The quality and characteristics of these activities might constitute as an interesting tool to analyze students' ability to generate ideas and assumptions using physics knowledge, which would reflect expectations from the social environment where these are created. Consequently, this analysis also provide interesting evidence regarding differences in instruction, emphasis on different concepts, as well as the situations where instructors are more comfortable introducing them.

\section{METHODS}

Data was collected in week 7 of the semester during a problem solving session from three sections of a mechanics course at a University in Chile, taught by three different instructors. The instructor of the Mixed section (S1) alternated each week between text book and ill-structured problems, whereas the Traditional section (S2) used only textbook problems. The Treatment section (S3) used weekly ill-structured problems. In weeks where Mixed section administered ill-structured problems, these were the same activities implemented in the Treatment section. In addition, the instructor of the Treatment section guided the session by encouraging students to make creative assumptions for their solutions, whereas instructors of the Mixed and Traditional sections did not. Participants were engineering students enrolled in a mechanics course $(32.1 \%$ female) that met three times a week for a semester. Student groups were consisted 
of 3-4 participants, for a total of 26 teams (Mixed $=9$; Traditional $=10$; Treatment $=7$ ). The task was framed as part of university activity for the community, where students needed to design physics problems using the principles of CM to help secondary students from a local high school better understand circular kinematics.

Two researchers analyzed the data, identifying the attributes and characteristics of the student generated problems. The first wave of coding problem characteristics was conducted independently by the lead author. Then, the first and second author revisited the coding and revised the coding scheme. After agreement, both researchers coded independently $40 \%$ of the data, obtaining a Cohen's Kappa of .92. In Table I we show the codes for the emergent problem characteristics, joined by their description. Analysis of these codes is based on their observed frequency per section. For graphical representation, problem characteristics such as type of information, number of questions and equations needed, contextual details, word count and cognitive demand were standardized to have a mean of 0.0 and standard deviation of 1.0, whereas we used raw frequency count for physics concepts asked.

\section{RESULTS}

\section{A. Physics problems}

Fig. 1 depicts samples of problems generated by groups from all three sections. All three problems provide contextual details as actions and/or subjects performing them (e.g., bus covering a distance, video game characters performing the action of tossing barrels, and bicycle moving while changing gears). In order to solve both problems, students would need to recall and recognize physics concepts, comprehend the type of information required, as well as represent concepts and principles in mathematical expressions [11]. Further, problem 3 includes an additional cognitive step of analysis that is implicitly embedded in the problem, and involves a comparison of tangential speed at different radius (gears) based on a constant angular speed (i.e., matching) [11].

Only problem 2 includes data that is ready to use (e.g., angular speed of $2 \pi$ and $10 \pi \mathrm{rad} / \mathrm{s}$; time $3 \mathrm{~s}$; time $5 \mathrm{~s}$.). All three activities require solvers convert measurement units (e.g., Problem 1. $2 \mathrm{~cm} / \mathrm{s}^{2}$ to $\mathrm{m} / \mathrm{s}^{2}$; Problem 2. units from $\mathrm{km}$ and $\mathrm{cm}$ to $\mathrm{m}$; radius from $\mathrm{cm}$ to $\mathrm{m}$; and Problem $3 . \mathrm{km} / \mathrm{h}$ to $\mathrm{m} / \mathrm{s}$ ). Only problem 3 requires solvers to research the radius for the different gears in the disc. Problems 1 and 3 also include information that requires translating text into math expressions, such as "the bus starts from rest" to $\omega_{0}=0 \mathrm{rad} / \mathrm{s}$, and to identify that the number of revolutions per second is the definition for frequency. Moreover, problems 1 and 3 also require algebraic treatment for linear to angular acceleration through $a_{t}=\alpha R$, and frequency $f$, into angular frequency $\omega$, through $\omega=2 \pi f$, respectively. Finally, problem 1 assumes that the position of interest is at the edge of the bus's
Problem 1 from Mixed Section:

A bus starts from rest after picking up passengers to take them to their destination $1.5 \mathrm{~km}$ from starting point. The wheels of the bus have an $80 \mathrm{~cm}$ of radius and this travels with a constant acceleration of $2 \mathrm{~cm} / \mathrm{s}^{2}$. With the above information respond: a) How long does it take for the bus to get to its final destination? b) What is the wheel's final angular speed? c) How many revolutions does the wheel complete to reach its final destination? d) What is the value of the tangential velocity?

Problem 2 from Traditional Section:

Donkey Kong wants to throw barrels to King $\mathrm{K}$ Rool. For this throws one with an angular speed of $2 \pi \mathrm{rad} / \mathrm{s}$. By knowing that at $3 \mathrm{~s}$ its speed is $10 \pi \mathrm{rad} / \mathrm{s}$, and that the barrel impacts at $5 \mathrm{~s}$, determine: $a$. the angle covered by the barrel; $b$. the magnitude of the centripetal and tangential acceleration at the moment of impact at $6 \mathrm{~cm}$ from its center; $c$. frequency and period.

Problem 3 from Treatment Section:

When a 7 gear bicycle travels a $20 \mathrm{~km} / \mathrm{h}$, wheels complete 2.94 cycles every second at 6 th gear, whereas at gear 1 they need 1.77 complete cycles per second to keep on the same speed. How many turns per second are necessary to maintain the same speed of $20 \mathrm{~km} / \mathrm{h}$ if one were to use every gear available in the bicycle?

FIG. 1. Sample of physics problems generated by students Mixed, Traditional and Treatment sections.

wheel, located at $\theta_{0}=0 \mathrm{rad}$, while, in problem 2 , the initial conditions are considered at time $3 \mathrm{~s}$, rather than $0 \mathrm{~s}$, with $\theta(3)=0 \mathrm{rad}$ is the initial angular position of interest in the barrel at $3 \mathrm{~s}$. In the face of this type of information (i.e., assumptions) one may recognize that students either omitted information as a way to challenge their audience or this occurred by accident and indicates a misconception about the nature of well-structured physics problems.

Because problems 1 and 2 (Fig. 1) are situations that entail accelerated CM, solvers might use both expression for $\theta(t)=\theta_{0}+\omega_{0} t+\frac{1}{2} \alpha t^{2}$ and $\omega(t)=\omega_{0}+\alpha t$. In problem 1 , a solver might utilize $\theta(t)$ to determine the total time spent on the bus ride, before adjusting the $1.5 \mathrm{~km}$ into angular distance (question c.). The final time will be useful to obtain the magnitude of the angular speed from $\omega(t)$, and subsequently $v_{f}$. For problem 2 the procedure is similar, but the time of the impact is given, while $\alpha=8 \pi / 3 \mathrm{rad} / \mathrm{s}^{2}$. Question a on problem 2 can be determine through $\theta(t)$, while questions $b$ and $c$ will require angular speed at the time of impact $(5 \mathrm{~s})$, to then calculate linear speed through $v_{t}=\omega R$, and finally use $a_{c}=v^{2} / R$ and $a_{t}=\alpha R$ for the magnitude for centripetal and tangential acceleration respectively. Question c on problem 2 may be confusing to solvers due to the accelerated motion of the barrels, and the change in frequency and period over time. One may interpret this as an error associated in the writing of the problem, or as a conceptual misunderstanding linked to the nature of CM. Finally, in order to solve problem 3 , solvers must understand the relationship between angular and tangential speed is given by $v_{t}=\omega R$. Here, the disc measures the same tangential speed $\left(v_{t}\right)$ every time a gear is changed (i.e., change in $R$ ), and therefore, in order to maintain the same tangential speed, the product $\omega R$ must be constant. Even though solvers may obtain radius for gears 6 and 1 , the question consists on determine the frequency for all the 7 different gears available in the bike. With this information, solvers can use the relationship between $v_{t}, \omega$ and $R$ to determine the angular speed at which each gear needs to spin for the bike to maintain a constant speed. 
TABLE I. Code description of problem characteristics.

\begin{tabular}{|c|c|}
\hline Code & Description \\
\hline Physics Concepts Asked & Physics concepts used as problem items (e.g., angular speed, tangential acceleration). \\
\hline \multicolumn{2}{|l|}{ Type of Information } \\
\hline Ready-to-Use Information & Data is explicitly presented in the problem and with appropriate units for its use. \\
\hline Conversion of Units & Physical quantities that need conversion to respect the IS of units (i.e., $\mathrm{m}$ and s). \\
\hline Text to Math Representations & $\begin{array}{l}\text { Physics information is presented in written form and needs translation into mathematical ex- } \\
\text { pressions (e.g., "begin its motion from rest" or "uniform motion"). }\end{array}$ \\
\hline Algebraic Transformation & Physics information for solving the problem needs algebraic steps for accessing and using it. \\
\hline Information Research & The problem requires researching appropriate magnitudes to solve the problem. \\
\hline Number of Physics Concepts Asked & Number of physics concepts used as problem items. \\
\hline Number of Equations Needed & Number of equations required to solve the problem. \\
\hline Contextual Details & Elements from real-life activities, and/or actors witnessing or engaging in actions. \\
\hline Word Count & Number of words used on the problems' description. \\
\hline Cognitive Demand & Taken from a taxonomy of introductory physics problems [11]. \\
\hline
\end{tabular}

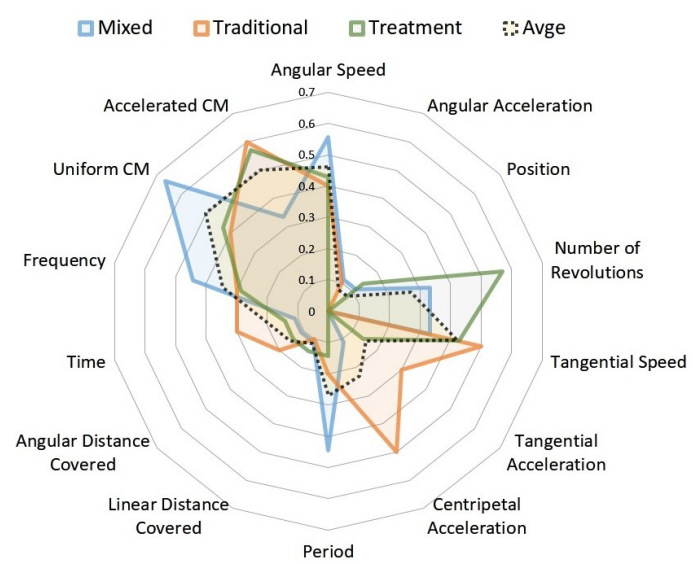

FIG. 2. Frequency of physics concepts used in student generated problems by sections.

\section{B. Physics Concepts}

The radial graph in Fig. 2 depicts that problems from all three sections display similar shapes, with higher observed variations for concepts such as number of revolutions $(\mathrm{S} 1=$ $0.3 ; \mathrm{S} 2=0.0 ; \mathrm{S} 3=0.6)$, centripetal acceleration $(\mathrm{S} 1=0.1$; $\mathrm{S} 2=0.5 ; \mathrm{S} 3=0.0)$, and period $(\mathrm{S} 1=0.4 ; \mathrm{S} 2=0.2 ; \mathrm{S} 3=$ $0.1)$. Both Traditional and Treatment sections showed similar tendency to create problems with acceleration (Accelerated $\mathrm{CM}$ ), while Mixed created problems that focused on constant angular speed (Uniform CM).

In the Mixed section, the most commonly used principle was angular speed $(n=5)$, while angular acceleration, position, centripetal acceleration, distance covered, angular distance and time were the least used $(n=1) .42 \%$ of the concepts included in the questions posed in this section relate to angular speed, period, and frequency, three concepts whose defi- nitions are intertwined through $\omega=2 \pi / T=2 \pi f$. For the Traditional section, tangential speed, centripetal acceleration and angular speed are the three most frequently used (48.2\%), whereas angular acceleration and linear distance are the least asked concepts. Time, angular speed, frequency and angular acceleration account for $55.17 \%$ of the information given. The tendency in this section was to ask information regarding different forms of speed and acceleration, thus implying multiple algebraic expressions such as tangential speed $v_{t}=\omega R$, with $\omega(t)$ in a constant acceleration motion.

In the Treatment section, the number of revolutions and angular and tangential speed accounted for $55.6 \%$ of the concepts used. Angular distance, time, period, tangential speed, position and frequency are only asked once, while angular and centripetal acceleration were never included. Here, angular distance and the number of revolutions are two directly connected magnitudes, as in order to know the number of revolutions completed, students must know either the linear distance covered, or the angular distance, and then divide by the perimeter or the angle of one revolution $(2 \pi)$ respectively. The most frequent concepts are time $(n=5,20 \%)$, tangential acceleration $(n=4,16 \%)$, angular acceleration $(n=3,12 \%)$, diameter and angular position $(n=3,12 \%$ each).

\section{Problem Characteristics}

As with the physics concepts, we aggregated the average score for problem characteristics by section. To ease interpretation of characteristics by section, Fig. 3 shows the standardized count over each coded variable, and includes Problem Elaboration, a new variable made from the section average of the of the standardized characteristics. The radial representation shows the areas that each section emphasized in their problems. Student groups from the Treatment section included, on average, more ready to use information (1.86) than 


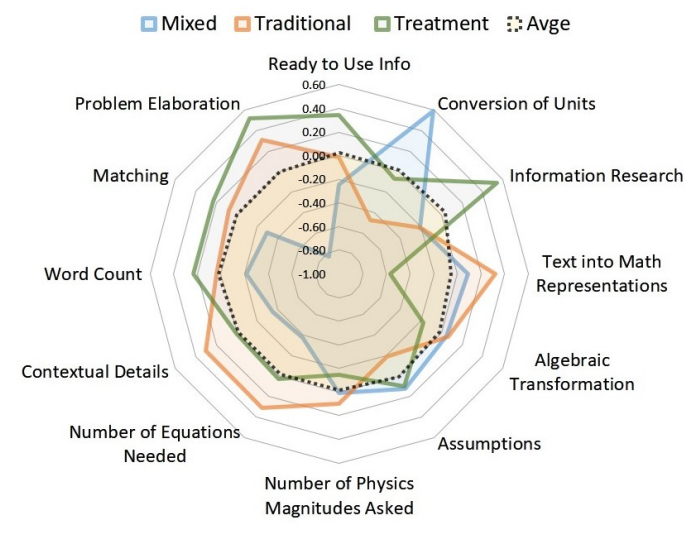

FIG. 3. Average problem characteristics by section.

students from the Mixed (1.1) and the Traditional (1.4). Similarly, conversion of units is more often used by groups from the Mixed section (1.4) than the others, whereas information research appears once in the Treatment section (problem 3, Fig. 1). Translating text into math representations was found more often on groups from Traditional section (0.9), followed by Mixed section (0.78). In addition, algebraic treatment and making assumptions are observed at fairly similar level across sections. On average, students from all sections created problems with more than 2 questions, requiring 3 equations for solutions. The highest mathematical demand was observed on problems from Traditional (3.4) and Treatment (3.14). Similarly, problems from the Traditional section added more contextual details (2.3) than the average. In terms of word count, the Treatment section (65 words) scored above the sample mean, while problems from the Mixed section were the shortest (51 words), often taking the form of: "A body spins with uniform $\mathrm{CM}$ and covers $1080^{\circ}$ in $15 \mathrm{~s}$. Calculate the angular speed".

For cognitive demand, all problems in the sample required recalling and recognizing information, comprehension of physics concepts, and symbolizing. Yet, only two problems were categorized as matching (i.e., compare physics magnitudes, quantities or phenomena), one on Traditional and Treatment. The strong difference in Matching observed on Fig. 3 between sections is an artifact of the average per class, due to the difference in the number of problems generated per section. Finally, we summed up the standardized score for each variable characteristic and calculated the mean per section, as a measure of overall elaboration, which might suggest the relative quality and complexity of the problems created on each section. Learning activities generated in Mixed section (-0.83) were the least elaborated, while problems from Traditional and Treatment showed a mean score of 0.31 and 0.52 respectively.

\section{CONCLUSIONS}

By exploring student generated problems, we identified the key concepts and attributes that characterize student (mis)conceptions of circular motion. In doing this, we show a novel way of assessing student generated problems, taking into account physics concepts and the different characteristics embedded in their problems. Students from all 3 sections showed adequate manipulation of the concepts for circular motion and applying them to creating a problem. The differences across sections on the use of concepts (e.g., uniform versus accelerated motion) suggest the tendency for some instructors to emphasize or repeat some concepts over others, as may be the case with the Mixed section when addressing a majority of situations with no acceleration, compared to problems from the other two sections. Limitations and areas of improvement relate to the speculation that assumptions as problem characteristics are not intentional, but caused by lack of knowledge. In-depth analysis of alternative sources of error on students' problems, as well as mechanisms on how to optimize problem generation for appropriate knowledge development are goals for future research.

All sections had characteristics in their generated problems that included assumptions, algebraic transformations, and similar numbers of physics magnitudes asked for. The Traditional and Treatment sections wrote problems that showed higher problem elaboration. It is a surprise that the Mixed section had lower elaboration than the Treatment section. Perhaps the learning goal of the task (i.e., design a physics problem for secondary students) may have motivated students in the Traditional section to utilize characteristics from textbook problems that were in their repository of activities to design problems in an effective way. The Mixed section worked on ill-structured problems, but the instructor did not emphasize the importance of assumptions in the face of illstructured activities. Consequently, highlighting the role of assumption making when addressing creative tasks we believe had positive effects over students' expectations and motivation for generating problems, as suggested by the high problem elaboration found on problems from Treatment section, whose instructor engaged in such a positive narrative for creativity. Finally, engaging on this creative activity contributes to students' experiences of generating ideas and assumptions, abilities needed for solving real world problems [1].

\section{ACKNOWLEDGMENTS}

This material is based upon work supported by the AAPT E. Leonard Jossem International Education Fund. Any opinions, findings, and conclusions or recommendations expressed in this material are those of the author(s) and do not necessarily reflect the views of the American Association of Physics Teachers. 
[1] D. Fortus, Science Education, 93, 1 (2008).

[2] R.K. Sawyer and S. DeZutter, Psych. Aesth. Creat. Arts, 3, 2, (2009).

[3] D.H. Jonassen, Ed. Tech. Res. and Dev. 48, 4 (2000).

[4] J.P. Mestre, Applied Dev. Psych. 23, (2002).

[5] J. Hardy et al, Int. J. Sc. Ed.36, 13, (2014).

[6] T.M. Amabile, Creativity in Context (Westview Press, Boulder,
1996).

[7] N. Shin et al, Journal R. Sc. Teach. 40, 1, (2003).

[8] T. Byun and G. Lee, Am. J. Phys. 82, (2014).

[9] I. Molenaar and M.M. Chiu, Small Group. Res. 48, 2 (2017).

[10] B.R. Johnson and C.J. D'Lauro, Small Group Res. 49, 2 (2018).

[11] R.E. Teodorescu et al, Phys. Rev. ST. Phys. Ed. Res. 9, (2013). 